\section{Neurotrophic tyrosine kinase gene fusions: another opportunity for targeting in lung cancer}
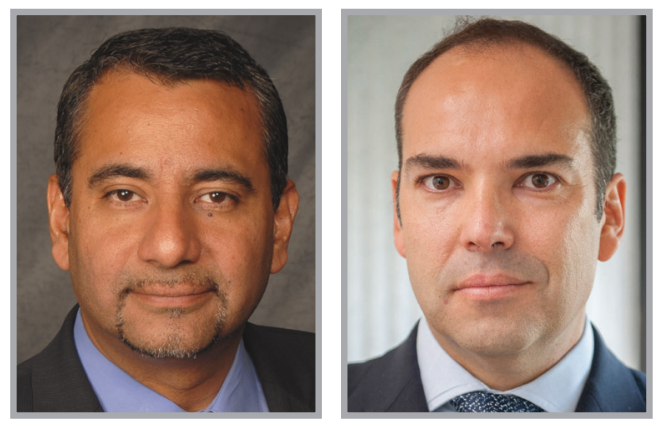

\author{
"New drugs are currently \\ under investigation to target \\ tumors harboring activating \\ NTRK molecular alterations in \\ early Phase I studies...”
}

Luis E Raez ${ }^{1,2}$ \& Christian Rolfo ${ }^{* 3}$

First draft submitted: 28 February 2016; Accepted for publication: 1 March 2016; Published online: 22 March 2016

\begin{abstract}
Neurotrophic tyrosine kinase gene fusions

Neurotrophic tyrosine kinase (NTRK) genes encode for the Trk-family proteins: TrkA (encoded by NTRK1), TrkB (encoded by NTRK2) and TrkC (encoded by NTRK3). These family proteins normally play a role in the development of the CNS and PNS [1]. They modulate cells growth, proliferation, repair, maintenance and apoptosis [2]. Lately, NTRK fusions have been found in solid tumors creating oncogenic fusions responsible for transformation of cancer cells, proliferation and survival [3]. Several studies have documented how the presence of these three gene fusions is associate with worse survivals in all types of lung cancers [4]. NTRK1 oncogenic fusions have been detected by FISH or next-generation sequencing (NGS) in about $3 \%$ of NSCLC, while NTRK 2 and NTRK3 fusions in about $1 \%$ of NSCLC including all types [3,5]. However, there are other two studies that found very
\end{abstract}

low numbers in patients with early stage NSCLC after tumor resection [6,7]. Also, we emphasize the presence of the fusion gene for its oncogenic potential because other alterations like point mutations can be found in these genes with no relevant oncogenic potential [5].

\section{Preclinical studies}

Entrectinib (RXDX-101) is a potent multikinase small molecule inhibitor, which selectively inhibits the tyrosine kinases NTRK1, NTRK2 and NTRK3, ROS1 and ALK as it has been proved in vitro and in vivo in several tumor models. In mice xenografts of colorectal cancer cells with the TrkA fusion TPM3-NTRK1, the treatment with entrectinib created tumor regression [8]. Also, it inhibited both in vitro and in vivo growth of $E M L-A L K / R O S 1$ rearranged NSCLC models, even crossing the blood-brain barrier in mice with NCI-H2228 EML4-ALK-driven lung cancer cells [9]. Other TRKA inhibitors

\section{KEYWORDS}

- lung cancer • NTRK inhibitors

- targeted therapy • TRK1/TRK2/TRK3 fusions

“...NTRK fusions have been found in solid tumors creating oncogenic fusions responsible for transformation of cancer cells, proliferation and survival." 
"NTRK fusions were discovered many years ago

but were not consider actionable oncogenic drivers until recently..." like CEP-701 and ARRY-470 also showed success against lung cancer cells expressing NTRK1 fusions [3]. LOXO-101 is an ATP-competitive pan-NTRK inhibitor that has shown antitumor activity against lung adenocarcinomas. The reported IC50 was $<100 \mathrm{nM} / 1$ for lung cancer cells and the inhibition of the autophosphorylation of TrkA fusion oncogenic protein and ERK1/2 was also observed both in lung and colorectal cancer cell lines [10]. In xenograft models injected with Trk-driven cell lines, treated with LOXO-101 a dose-dependent tumor growth inhibition was observed [10]. Another compound AZD7451 was tested on colorectal cancer cell lines harboring TPM-NTRK1 fusion (KM12 cell line) and the H460 (large cell neuroendocrine carcinoma [LCNEC] cell line exhibiting NTRK2 expression). The cell lines (KM12 and $\mathrm{H} 460$ ) have their proliferation inhibited. Moreover, an analysis of the H460 cells demonstrated decreased levels of phosphorylation of NTRK2 after AZD7451 exposure [6]. These and other preclinical studies in colorectal cell lines suggested a potential for targeting NRTKdependent cancer cell populations due to the fact that these gene fusions are oncogenic drivers of the cancer tumors.

\section{Clinical trials with NTRK inhibitors}

Entrectinib first showed activity in colorectal cancer harboring a TPM3-NTRK1 fusions [8]. The Phase I studies ALKA-372-001 and STARTRK-1 were dose-escalation trials of entrectinib for patients with advanced or metastatic solid tumors harboring TrkA, ROS1 or ALK molecular alterations. The results of both trials, involving the first 67 patients enrolled (of whom 38 were NSCLC), were presented at the 2015 American Society of Clinical Oncology [11,12]. In total, 11 patients received the recommended Phase II dose of entrectinib and ten responses were reported; and three out of three patients harboring NTRK translocations obtained a partial response (PR). In total, nine out of 11 patients obtained durable responses for up to 16 cycles and remained on treatment. Entrectenib was well tolerated with the most common side effects: fatigue (33\%), dysgeusia $(26 \%)$, constipation $(22 \%)$, nausea $(22 \%)$ and paresthesia $(22 \%)$. Three treatmentrelated grade 3 adverse events were reported: neutropenia (one patient), asthenia (one patient) and muscle weakness (one patient), all of which resolved after dose reduction. Two dose-limiting toxicities at a daily dose of $800 \mathrm{mg}$ were reported: cognitive impairment and fatigue; both resolved upon study of drug interruption. The recommended Phase II dose was $400 \mathrm{mg} / \mathrm{m}^{2}$ per day. In another publication, Farago et al. [7] screened 1378 cases of NSCLC using PCR and doing confirmation by FISH for the presence of NTRK1 gene rearrangements. Two patients $(0.1 \%)$ were found to have NTRK1 gene rearrangements: one was a TPM3-NTRK1 rearrangement, and in the second case, a fusion transcript containing sequence from SQSTM1 (sequestosome 1, which has previously been described as a fusion partner with $A L K$ in NSCLC) and NTRK1 was observed. The patient presenting the SQSTM1-NTRK1 fusion participated in a Phase I clinical trial with entrectinib, in the $400 \mathrm{mg} / \mathrm{m}^{2}$ dose cohort, he did not present any grade 3-4 adverse events. Clinical benefit was obtained after 3 weeks of treatment (improvement in dyspnea and pain), and he has continued on treatment for $>6$ months with an ongoing PR. More interestingly, this patient presented brain metastases at the moment of study entry, and a complete response in the CNS was observed after 155 days of the study treatment, suggesting entrectinib can effectively cross the blood-brain barrier and be effective in CNS metastases treatment [7]. STARTRK-2 trial (NCT02568267) is a Phase II basket study evaluating entrectinib for the treatment of solid tumors harboring $N T R K 1 / 2 / 3, R O S 1$ or $A L K$ gene rearrangements currently recruiting patients in the USA.

LOXO-101 is an ATP-competitive pan-Trk inhibitor, which has demonstrated encouraging activity on preclinical studies. A Phase I trial (NCT02122913) is currently recruiting patients [13]. The study has enrolled six patients with NTRK fusions, including one NSCLC (TPR-NTRK1), two mammary analog secretory carcinoma (ETV6-NTRK3), one thyroid carcinoma (ETV6-NTRK3), one gastrointestinal stromal tumors (GIST; ETV6-NTRK3) and one soft tissue sarcoma (STS; LMNANTRK1). All three patients evaluable for efficacy (STS, mammary analog secretory carcinoma and GIST) reported partial responses to LOXO-101 within 3-4 months (overall response rate [ORR]: $100 \%$ ), and all patients currently remain on study without progression at the time of the report. No study drug-related severe adverse events (SAEs) have been reported and the maximum tolerated dose (MTD) has not been yet defined. LOXO-101 is well-tolerated, with most common adverse events including 
grade 1/2 fatigue, dizziness, anemia and nausea [14]. Doebele et al. published a case report reporting a PR and symptom improvement in a patient with a metastatic STS harboring the $L M N A-N T R K 1$ gene fusion, participating in the LOXO-101 Phase I trial [10]. A Phase II basket study of LOXO-101 in patients with NTRK fusion positive solid tumors, including CNS tumors, has enrolled the first patient on October 2015 (NCT02576431) and its preliminary results are expected to be available by April 2018.

New drugs are currently under investigation to target tumors harboring activating NTRK molecular alterations in early Phase I studies including patients with advanced solid tumors like the multitarget inhibitors XL-184, MGCD516 and DCC-2701 that are under evaluation in advanced cancers positive for different molecular alterations, including NTRK (NCT02219711, NCT02228811). Among these, DCC-2701 (altiratinib), a MET, TIE2, VEGFR2 and TRK kinase inhibitor currently in Phase I clinical development has recently received Orphan Drug Designation for glioblastoma by the US FDA. AZD7451 is a tyrosine kinase inhibitor that selectively targets NTRK1, NTRK2 and NTRK3 kinases, being considered then a pan NTRK kinase inhibitor, which has shown promising preclinical activity in NTRK1 fusion positive lung cancer cell lines [6]. Finally, we have the TrkA/ALK inhibitor TSR-011 (NCT02048488), the pan-Trk/ROS1 inhibitor DS6051b (NCT02279433) and the pan-Trk inhibitor PLX-7486 (NCT01804530).

\section{What we expect from NTRK inhibitors?}

NTRK fusions were discovered many years ago but were not consider actionable oncogenic drivers until recently when the preclinical and early Phase I studies showed activity in colorectal cancer and NSCLC. Maybe to say that the NTRK fusions are present in $<5 \%$ of the NSCLC might sound not very exciting unless we remember that lung cancers are expected to be in $>225,000$ new patients in 2016. This means that we are potentially developing a new therapy for $>12,000$ patients that did not have options before. As we know so far the NTRK fusion seems to be exclusive of other molecular alterations in the $E G F R$ or $A L K$ genes, so the only option for these patients now a days is palliative chemotherapy. Entrectinib and LOXO-101 represent two new drugs that are pan-Trk inhibitors have shown promising activity and good tolerability profile in Phase I trials of advanced solid tumors and NSCLC harboring NTRK rearrangements. On the basis of such promissory results, entrectinib has already received the Orphan Drug Designation by FDA for the treatment of NTRK, $A L K$ and ROS1-positive advanced NSCLC.

Also more than ever we agree that the new concept of 'basket trials' is absolutely necessary not only for the Phase II of these compounds but for other solid tumors that might harbor these NTRK fusions and can benefit sooner than later with these inhibitors instead of expend many years of lengthens single tumor studies. It is our hope that if the current Phase II trials confirm the activity and low toxicity profile of these compounds we can have them available sooner rather than later to the patients as the FDA has done before with crizotinib and other drugs that have conditional approval. Of course with all new discoveries there are more questions than answers at the beginning, we do not want to be over optimistic, and these drugs have to be verified in the Phase II and III trials and the toxicity profile corroborated with long-term follow-up.

Another potential problem is the fact that the best way to detect the oncogenic fusions is by FISH or NGS; if we consider that currently we recommend FISH for ALK and ROS-1 fusions in NSCLC patients, soon we might have to recommend five FISH tests for every new NSCLC patient (ALK, ROS-1, NTRK 1/2/3), something that increases the costs of the testing. Then the alternative for several people is to do NGS for all gene fusions; this is in discussion because despite the fact that we can do NGS for ALK, the current standard is still FISH. The other more attractive options are to validate immunohistochemistry for ALK and ROS-1 and hopefully do the same 1 day for the NTRK1/2/3 oncogenic fusions, making much easier the testing at the level of local hospitals. The last option for the fans of PCR is to validate this technique to detect these alterations. All of these technologies have pros and cons, and we can complicate this more by mentioning that we no longer have to worry only to find these oncogenic drivers in tissue, but now we can do in blood (liquid biopsies) with the same techniques we mentioned before when we isolate DNA from tumor cells or plasma.

We conclude that in the short term, we might have another generation of compounds that target the NTRK oncogenic fusions that will benefit a significant number of NSCLC patients
"New drugs are currently under investigation to target tumors harboring activating NTRK molecular alterations...” 
that currently do not have other options than palliative chemotherapy.

Financial \& competing interests disclosure

LE Raez has research support from LOXO oncology. The authors have no other relevant affiliations or financial involvement with any organization or entity with a financial interest in or financial conflict with the subject matter or materials discussed in the manuscript apart from those disclosed.

No writing assistance was utilized in the production of this manuscript.

\section{References}

1 Brodeur GM, Minturn JE, Ho R et al. Trk receptor expression and inhibition in neuroblastomas. Clin. Cancer Res. 15, 3244-3250 (2009).

2 Kaplan DR, Martin-Zanca D, Parada LF. Tyrosine phosphorylation and tyrosine kinase activity of the trk proto-oncogene product induced by NGF. Nature 350, 158-160 (1991)

3 Vaishnavi A, Cappelletti M, Le AT et al. Oncogenic and drug-sensitive NTRK1 rearrangements in lung cancer. Nat. Med. 19, 1469-1472 (2013).

4 Okamura K, Harada T, Wang $\mathrm{S}$ et al. Expression of TrkB and BDNF is associated with poor prognosis in non-small cell lung cancer. Lung Cancer 78, 100-106 (2012).

5 Harada T, Yatabe $\mathrm{Y}$, Takeshita $\mathrm{M}$ et al. Role and relevance of TrkB mutations and expression in non-small cell lung cancer. Clin. Cancer Res. 17, 2638-2645 (2011).

6 Tatematsu T, Sasaki H, Shimizu S et al. Investigation of neurotrophic tyrosine kinase receptor 1 fusions and neurotrophic tyrosine kinase receptor family expression in non-small-cell lung cancer and sensitivity to AZD7451 in vitro. Mol. Clin. Oncol. 2, 725-730 (2014).

7 Farago AF, Le LP, Zheng Z et al. Durable clinical response to entrectinib in NTRK1rearranged non-small cell lung cancer. J. Thorac. Oncol. 10(12), 1670-1674 (2015).

8 Anderson D, Ciomei M, Banfi P et al. Inhibition of Trk-driven tumors by the pan-Trk inhibitor RXDX-101. Eur. J. Cancer 50(Suppl. 6), 101 (2014).

9 Ardini E, Menichincheri M, De Ponti C et al. Characterization of NMS-E628, a small molecule inhibitor of anaplastic lymphoma kinase with antitumor efficacy in ALKdependent lymphoma and non-small cell lung cancer models. Mol. Cancer Ther. 8 (12 Suppl.), Abstract A243 (2009).

10 Doebele RC, Davis LE, Vaishnavi A et al. An oncogenic NTRK fusion in a patient with soft-tissue sarcoma with response to the tropomyosin-related kinase inhibitor
LOXO-101. Cancer Discov. 5, 1049-1057 (2015).

11 De Braud FG, Niger M, Damian S et al. Alka-372-001: first-in-human, Phase I study of entrectinib - an oral pan-trk, ROS1, and ALK inhibitor - in patients with advanced solid tumors with relevant molecular alterations. J. Clin. Oncol. 33(Suppl.), Abstract 2517 (2015).

12 Patel MR, Bauer TM, Liu SV et al. STARTRK-1: Phase 1/2a study of entrectinib, an oral Pan-Trk, ROS1, and ALK inhibitor, in patients with advanced solid tumors with relevant molecular alterations. J. Clin. Oncol. 33(Suppl.), Abstract 2596 (2015).

13 Burris HA, Brose MS, Shaw AT et al. A first-in-human study of LOXO-101, a highly selective inhibitor of the tropomyosin receptor kinase (TRK) family. J. Clin. Oncol. 33(Suppl.), Abstract TPS2624 (2015).

14 TRK inhibitor shows early promise. Cancer Discov. doi:10.1158/2159-8290.CDNB2015-165 (2015) (Epub ahead of print). 